\title{
Aspectos reprodutivos do cangati, Parauchenipterus galeatus Linnaeus (Osteichthyes, Auchenipteridae) da Lagoa de Extremoz, Rio Grande do Norte, Brasil
}

\author{
Ana Patrícia T. de Medeiros, Naithirithi T. Chellappa \& Sathyabama Chellappa
}

Programa de Pós-Graduação em Bioecologia Aquática, Departamento de Oceanografia e Limnologia, Centro de Biociências, Universidade Federal do Rio Grande do Norte. Praia de Mãe Luiza, 59014-100 Natal, Rio Grande do Norte, Brasil. E-mail: anapatriciatargino@yahoo.com.br; bama@dol.ufrn.br

\begin{abstract}
Reproductive aspects of cangati, Parauchenipterus galeatus Linnaeus (Osteichthyes, Auchenipteridae) from the Extremoz Lake, Rio Grande do Norte, Brazil. The Lake Extremoz is one of the three big lakes in the State of Rio Grande do Norte. The present work was carried out in this lake during the period of October 2000 to September 2001, which included the dry and the rainy seasons. Parauchenipterus galeatus Linnaeus, 1766 represent $10 \%$ of the commercially important species captured from this lake. Fish samples were collected on a monthly basis, besides registering the rainfall, temperature, and dissolved oxygen of the lake. The populational structure of cangati and the stage of maturation of the gonads were determined, besides the gonadosomatic relation. The results indicate that the study population presented higher mean values of total length and weight during the dry season. Mature fishes were caught during the months of May, June and July which represented the reproductive season.
\end{abstract}

KEY WORDS. Gonadal maturation, gonadosomatic relation, population structure, reproductive season.

A ictiofauna da região compreendida no "polígono das secas" caracteriza-se por um reduzido número de espécies, uma pequena densidade populacional e um baixo endemismo. As poucas espécies existentes nos ecossistemas lênticos do Rio Grande do Norte tais como piranha, Serrasalmus nattereri Kner, 1858, pirambeba, Serrasalmus rhombeus Linnaeus, 1766 e muçum, Synbranchus marmoratus Bloch, 1795, eram aquelas que em épocas de estiagem sobreviviam em pequenos poços de água resultantes de pouca chuva (AвReu \& SiLva 1987). Por esse motivo iniciaram-se estudos limnológicos nos açudes, lagos e rios nordestinos, objetivando a introdução de espécies ícticas originárias de outras regiões, uma vez que a quantidade de peixes existentes não era bastante variada para aumentar significantemente a produção pesqueira (MARQues \& GURGEL 2000, CÂMARA et al. 2002). A Lagoa de Extremoz, situada numa região industrial do município de Extremoz destaca-se entre os três grandes lagos do Estado do Rio Grande do Norte, pela sua importância para o abastecimento da cidade de Natal e pela sua produção pesqueira (ARAújo et al. 2000).

O cangati, Parauchenipterus galeatus Linnaeus, 1766, é uma espécie amplamente distribuída na América do Sul. Ocorre geralmente nas áreas de matas alagadas e sob vegetação aquática flutuante e possui hábitos tipicamente noturnos (Borges et al. 1999). O presente trabalho teve como objetivo estudar os aspectos reprodutivos de $P$. galeatus na Lagoa de Extremoz, re- lacionando com variações climatológicas e físico-químicas tais como: pluviosidade, temperatura da água e oxigênio dissolvido.

\section{MATERIAL E MÉTODOS}

As coletas foram realizadas mensalmente na Lagoa de Extremoz, durante o período de outubro de 2000 a setembro de 2001, utilizando-se rede de espera de diferentes tamanhos e malhas. Os dados de precipitação pluviométrica, foram obtidos do Departamento de Meteorologia e Recursos Hídricos da EMPARN (Empresa de Pesquisa Agropecuária do Rio Grande do Norte). As estações de seca e chuva foram caracterizadas pelos dados de pluviosidade apresentados durante o período de estudo. Foram medidas as variáveis físico-químicas da água como a temperatura (através de um termômetro INCOTERM com filamento de mercúrio de mínima e máxima $\left(0-50^{\circ} \mathrm{C}\right) \mathrm{com}$ precisão de $\pm 0,1^{\circ} \mathrm{C}$ ) e o oxigênio dissolvido (através de um oxímetro YSI Model 51B).

Foram coletados 144 exemplares que foram medidos $(\mathrm{mm})$ e pesados $(\mathrm{g})$, e os estádios de maturação gonadal e a relação gonadossomática (RGS) avaliados. Para a RGS utilizouse a seguinte fórmula: $\mathrm{RGS}=(\mathrm{Wg} / \mathrm{Wc}) \mathrm{x} 100$, onde $\mathrm{Wg}=$ peso das gônadas $(\mathrm{g})$ e $\mathrm{Wc}=$ peso total do corpo menos o peso das gônadas (g) (VAzZOLER 1996). 


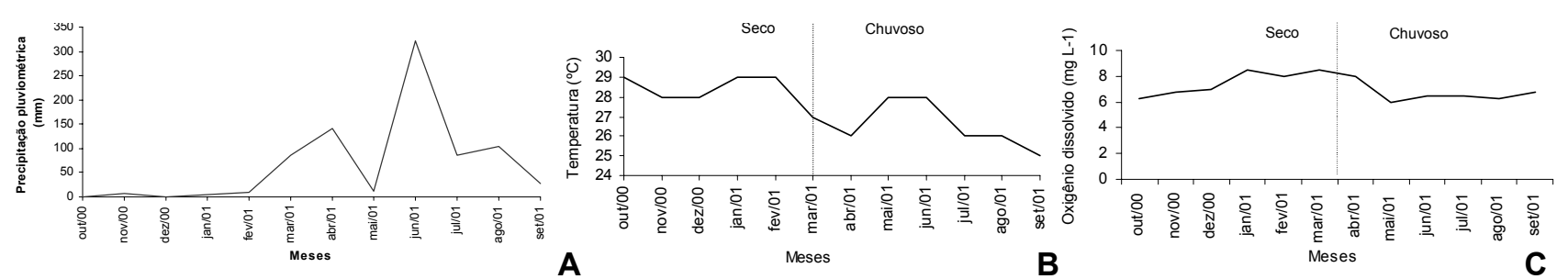

Figura 1. Valores mensais de precipitação pluviométrica (A), temperatura (B) e oxigênio dissolvido (C) da água superficial da Lagoa de Extremoz, durante o período de outubro de 2000 a setembro 2001.
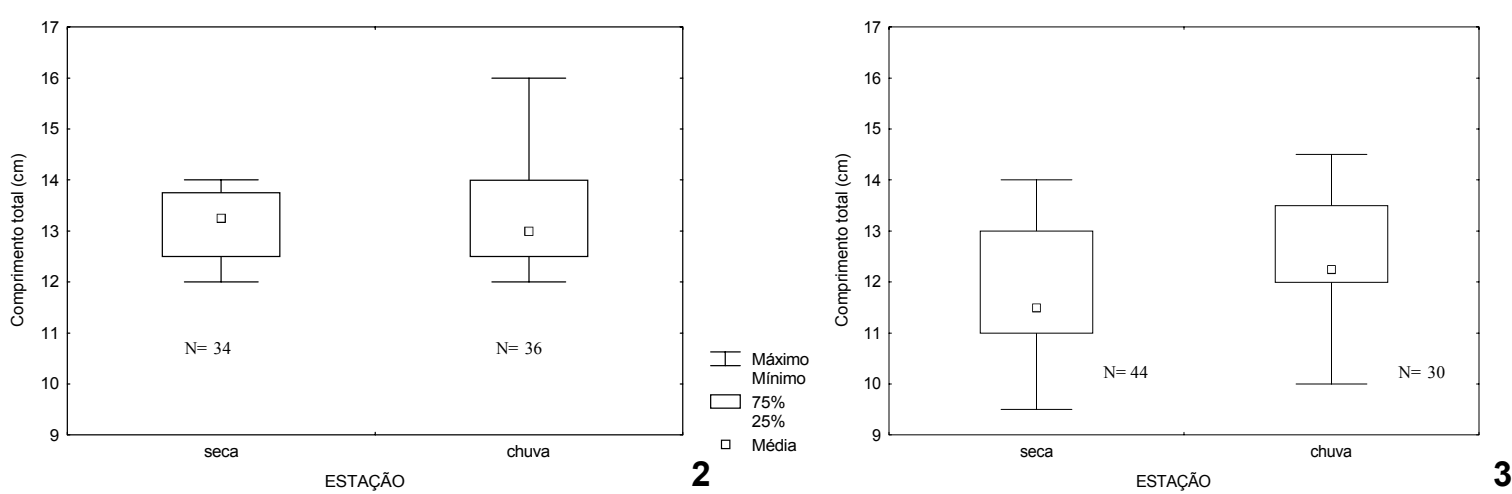

Figuras 2-3. Variação de amplitude e média do comprimento total de fêmeas (2) e de machos (3) de Parauchenipterus galeatus durante as estações seca (outubro de 2000 a fevereiro 2001) e chuvosa (março a julho de 2001).
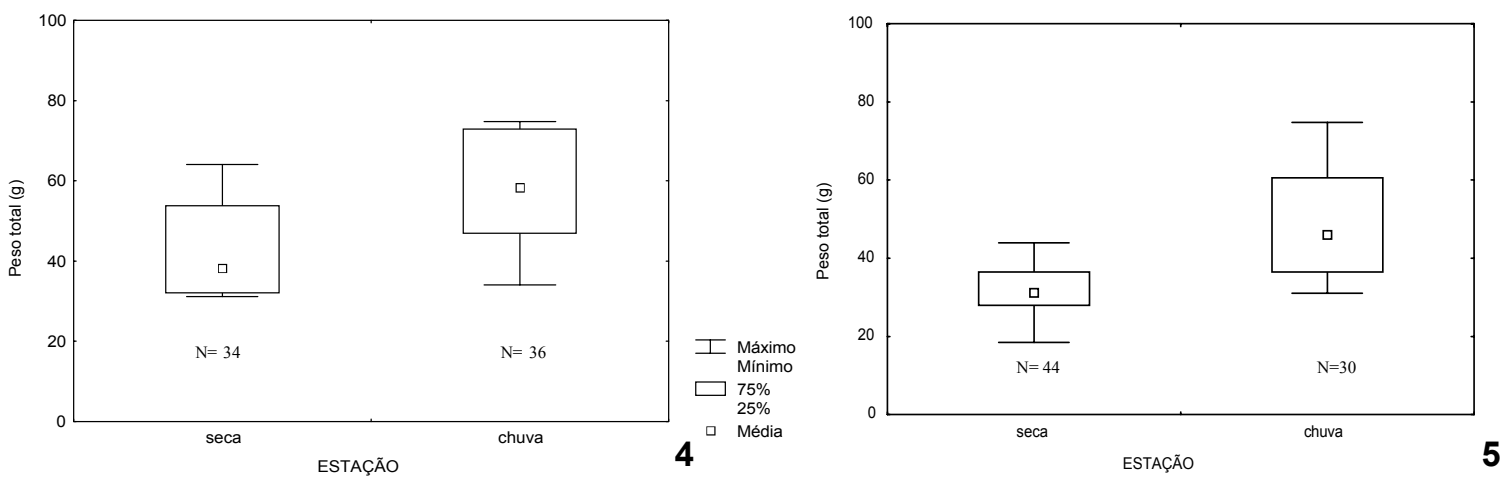

Figuras 4-5. Variação de amplitude e média do peso total de fêmeas (4) e de machos (5) de Parauchenipterus galeatus durante a estação seca (outubro de 2000 a fevereiro 2001) e chuvosa (março a julho de 2001).

\section{RESULTADOS}

Os dados pluviométricos sugerem que a área onde está localizada a Lagoa de Extremoz apresenta uma baixa escala de pluviosidade. Os meses de outubro de 2000 a fevereiro 2001 constituíram a estação seca com uma pluviosidade média de 4,2 mm e de março a setembro 2001, a estação chuvosa, com uma pluviosidade média de $137 \mathrm{~mm}$ (Fig. 1A).

A temperatura média anual da água da lagoa foi de $28^{\circ} \mathrm{C}$, com maiores valores ocorrendo no período seco, com média de $28,6^{\circ} \mathrm{C}$, e os menores no período de chuvas, com média de $27^{\circ} \mathrm{C}$ (Fig. 1B).
A média anual do oxigênio dissolvido foi de $7,2 \mathrm{mg} \mathrm{L}^{-1}$. Durante o período seco, os valores atingiram uma média de $7,3 \mathrm{mg} \mathrm{L}^{-1}$, enquanto que no período chuvoso a média foi de $7,1 \mathrm{mg} \mathrm{L}^{-1}$. Houve dois picos de oxigênio dissolvido, os quais foram de $8,5 \mathrm{mg} \mathrm{L}^{-1}$, em janeiro e em março de 2001, considerado período final da seca e inicial de chuvas respectivamente (Fig. 1C).

Foram coletados 144 exemplares do cangati (70 fêmeas e 74 machos) sendo a proporção sexual de aproximadamente $1: 1$. Durante a estação seca foram capturados 44 machos e 34 fêmeas, na estação chuvosa foram capturados 30 machos e 36 fêmeas. Observou-se que $P$. galeatus apresentou maior média

Revista Brasileira de Zoologia 20 (4): 647-650, dezembro 2003 

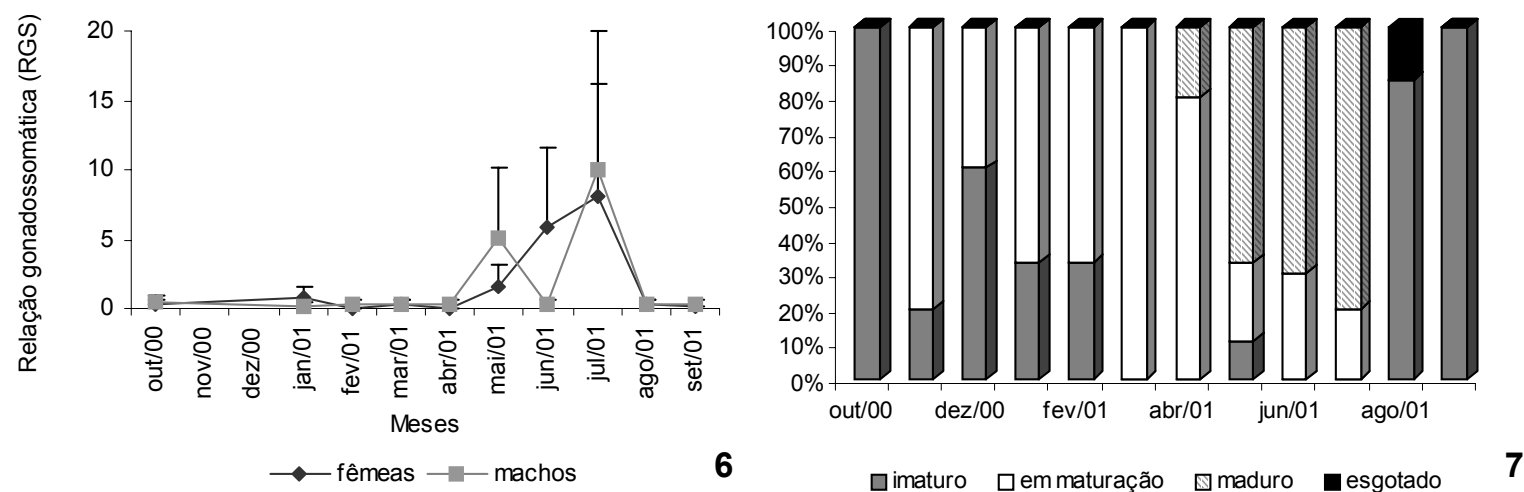

Figuras 6-7. (6) Média da Relação Gonadossomática (RGS) com desvio padrão para fêmeas e machos de Parauchenipterus galeatus durante o período de outubro de 2000 a setembro 2001; (7) distribuição mensal das freqüências relativas dos estádios de maturação gonadal de Parauchenipterus galeatus.

Tabela I. Estágios de maturação gonadal para machos e fêmeas de cangati, Parauchenipterus galeatus.

Estágios Machos Fêmeas

\begin{tabular}{|c|c|c|}
\hline Imaturo & Testículos filiformes, translúcidos, não vascularizados & $\begin{array}{l}\text { Ovários filiformes, translúcidos, sem presença de ovócitos visíveis a } \\
\text { olho nú }\end{array}$ \\
\hline m maturação & Testículos filiformes, branco-leitosos e vascularizados & $\begin{array}{l}\text { Ovários maiores, ocupando } 1 / 3 \text { a 2/3 da cavidade celomática, bem } \\
\text { vascularizados }\end{array}$ \\
\hline Maduro & $\begin{array}{l}\text { Testículos túrgidos, branco-leitosos, bem vasculariza- } \\
\text { dos, com fluência do líquido espermático a uma leve } \\
\text { pressão }\end{array}$ & $\begin{array}{l}\text { naior } \\
\text { ho }\end{array}$ \\
\hline Esvaziado & Testículos flácidos, com áreas hemorrágicas & $\begin{array}{l}\text { Ovários flácidos, presença de poucos ovócitos de tamanho pequeno, } \\
\text { com áreas hemorrágicas }\end{array}$ \\
\hline
\end{tabular}

de comprimento total e peso total durante a estação chuvosa, representando a fase de crescimento e o período inicial de maturação gonadal (Figs 2-5).

Os valores médios de RGS (relação gonadossomática) dos machos do cangati variaram de 0,3 na estação seca a 10 na estação chuvosa, enquanto as fêmeas apresentaram valores médios que variaram de 0,4 na estação seca a 8,1 na estação chuvosa (Fig. 6).

Foram registrados quatro estádios de maturação gonadal para $P$. galeatus: imaturo, em maturação, maduro e esgotado (Tab. I). No mês de outubro de 2000 , cem por cento dos indivíduos amostrados $(\mathrm{N}=11)$, se encontravam no estádio imaturo enquanto nos meses de janeiro e fevereiro de 2001, dois terços da amostra $(\mathrm{N}=7)$ se encontravam nesse estádio. Em março 2001 todos os indivíduos ( $\mathrm{N}=11)$ estavam em maturação, e no mês de abril $80 \%$ dos indivíduos capturados $(\mathrm{N}=9)$ se encontravam ainda em maturação, estando os vinte por cento restantes em estádio maduro. Nos meses de maio $(\mathrm{N}=8)$ e junho $(\mathrm{N}=10)$ os indivíduos maduros foram mais de sessenta por cento. O mês de agosto 2001 apresentou um terço dos indivíduos capturados $(\mathrm{N}=5)$ no estádio esgotado e o restante imaturos (Fig. 7).

\section{DISCUSSÃO}

O estádio de maturação gonadal e a relação gonadossomática, bem como os valores de comprimento total e de peso total, proporcionaram o conhecimento de alguns aspectos da reprodução de Parauchenipterus galeatus.

Os resultados obtidos neste trabalho indicaram um período reprodutivo prolongado que iniciou-se em maio e estendeu-se até agosto, coincidindo com a época chuvosa da região. Os indivíduos imaturos capturados no mês de agosto 2001 foram oriundos das atividades reprodutivas de adultos dos meses anteriores.

As variáveis comprimento total e peso total estão sob influência de diversos fatores, como densidade populacional, disponibilidade de alimento, $\mathrm{pH}$, temperatura e oxigênio, que por meio de interações existentes entre eles, poderão afetar os valores estimados das variáveis da relação (SuAssuna 1999). No presente estudo, foi observado que o cangati apresentou maior média de comprimento total e peso total durante a estação chuvosa, representando a fase de crescimento e o período inicial de maturação gonadal.

A proporção sexual observada para espécie em estudo não difere significantemente da esperada (1:1). Os dados obti-

Revista Brasileira de Zoologia 20 (4): 647-650, dezembro 2003 
dos estão de acordo com os encontrados por Borges et al. (1999) trabalhando com esta espécie, na Lagoa do Jiqui, município de Nísia Floresta, Rio Grande do Norte.

Os valores de RGS das fêmeas mostraram-se elevados no mês junho, enquanto que os o dos machos mostraram-se elevados no mês julho, coincidindo com a época chuvosa.

O aumento da precipitação durante a estação chuvosa provoca o aumento da vazão, possibilitando um incremento no volume d'água da lagoa. A pluviosidade, a temperatura da água (menores valores registrados durante a época chuvosa) e oxigênio dissolvido demonstraram maior relação com a época de reprodução da espécie estudada.

Parauchenipterus galeatus apresentou-se bem adaptado às condições ecológicas da lagoa, utilizando-a como habitat reprodutivo. O período reprodutivo coincide com o período chuvoso.

\section{AGRADECIMENTOS}

A primeira autora agradece a CAPES/MEC pela bolsa concedida durante o período de estudo.

\section{REFERÊNCIAS BIBLIOGRÁFICAS}

Abreu, U.L.B \& J.W.B. SiLVA. 1987. Análise da produção pesqueira em cinco açudes públicos administrados pelo Departamento Nacional de Obras Contra Seca (DNOCS), período
1966 a 1985. Boletim Técnico do DNOCS, Fortaleza, 45 (1/2): 27-50.

Araújo, M.F.F; I.A.S. Costa \& N.T. Chellappa. 2000. Comunidade Fitoplanctonica e variáveis ambientais na Lagoa de Extremoz, Natal-RN. Brasil. Acta Limnológica Brasiliensia, Belo Horizonte, 12, 127-140.

Borges S.A.G.V; H.C.B Gurgel \& B. Canan. 1999. Estrutura populacional de Parauchenipterus galeatus Linnaeus, 1766 (Siluriformes, Auchenipteridae), da Lagoa de Jiqui, Parnamirim, Rio Grande do Norte. Revista CERES, Viçosa, 46 (264): 209-218.

Câmara, M.R; N.T. Chellappa \& S. Chellappa. 2002. Ecologia reprodutiva do Cichla monoculus, um ciclídeo amazônico no semi-árido do Rio Grande do Norte. Acta Limnologica Brasiliensia, Belo Horizonte, 14 (2): 9-16.

Marques, D.K.S.; I.L. Rosa \&. H.C.B Gurgel 2000. Descrição histológica de gônadas de traíra, Hoplias malabaricus (Bloch) (Osteichthyes, Erytrinidae) da barragem do Rio Gramame, Alhandra, Paraíba, Brasil. Revista Brasileira de Zoologia, Curitiba, 17 (3): 573-582.

SuASSUNA, J. 1999. Contribuição ao estudo hidrológico do semiárido nordestino. Recife, Fundação Joaquim Nabuco, 62p.

VAzzoler, A.E.A.M. 1996. Biologia e reprodução de peixes teleósteos: teoria e prática. Maringá, Editora Universidade Estadual de Maringá, 169p.

Recebido em 11.XI.2002; aceito em 28.X.2003.

Revista Brasileira de Zoologia 20 (4): 647-650, dezembro 2003 